\title{
La Agricultura familiar en Santa Cruz, Patagonia Argentina: Caracterización de los núcleos agrícolas familiares
}

\section{Family Farming in Santa Cruz, Patagonia Argentina: Characterization of family agricultural nuclei}

\author{
Jorge Alberto Birgi ${ }^{1,2}$, Pablo Luis Peri ${ }^{1,2,3}$, Marcos Mauricio Ceconello ${ }^{1,2,4}$, Verónica \\ Beatriz Gargaglione ${ }^{1,2}$ \\ birgi.jorge@inta.gob.ar,peri.pablo@inta.gob.ar,ceconello.marcos@inta.gob.ar, \\ gargaglione.veronica@inta.gob.ar \\ ${ }^{1}$ Instituto Nacional de Tecnología Agropecuaria (INTA). \\ ${ }^{2}$ Universidad Nacional de la Patagonia Austral, Instituto Ciencias del Ambiente, \\ Sustentabilidad y Recursos Naturales (ICASUR), Río Gallegos. \\ ${ }^{3}$ Consejo Nacional del Investigaciones Científicas y Técnicas (CONICET). \\ ${ }^{4}$ Universidad Nacional de Tucumán. Facultad de Agronomía y Zootecnia, San Miguel de \\ Tucumán.
}

Recibido: 12/02/2020. Aceptado: 11/09/2020

\section{RESUMEN}

La agricultura familiar abarca a un grupo grande de personas que tienen distintos orígenes y una amplia diversidad cultural, pero que mantienen en común la producción de alimentos como forma de vida. El objetivo del presente trabajo fue caracterizar a la agricultura familiar de la provincia de Santa Cruz, determinando aspectos cualitativos, productivos y condiciones de producción de los núcleos de agricultura familiar. Se estudió el sector agrícola familiar de la provincia utilizando como base el análisis de 258 planillas del Registro Nacional de Agricultura Familiar relevadas durante los años 2014-2016. De dicho análisis surge que el tamaño medio de los predios en Santa Cruz es de 5 ha, se dedican principalmente a la agricultura y a la producción pecuaria, con predominio de la avicultura. En general, los productores realizan diversas actividades productivas en simultáneo. Se destaca por superficie implantada, la producción de forrajes, frutales, frutas finas y hortícolas de hoja. El presente trabajo es el primero en visibilizar numerosos aspectos de la agricultura familiar en Santa Cruz y presenta información inédita acerca de los núcleos de agricultura familiar en la provincia, contemplando a muchos productores que no figuran como tales en otros relevamientos como ser el Censo Agropecuario Nacional. Contar con este tipo de información resulta fundamental para las instituciones y los diversos actores sociales a fin de de monitorear su evolución en el tiempo y desarrollar políticas para su desarrollo y fortalecimiento.

Palabras clave: desarrollo rural; producción periurbana.

\begin{abstract}
Family farming encompasses a large group of people who have different origins and broad cultural diversity, but who keep food production in common as a way of life. The aim of this study was to characterize family farming in the province of Santa Cruz, determining qualitative and productive aspects and also production conditions of the family farming
\end{abstract}


nucleus. The family agricultural sector of the province was studied using the analysis of 258 forms of the National Registry of Family Agriculture surveyed during the years 2014-2016. From this analysis it appears that the average size of the farms in Santa Cruz is 5 ha, they are mainly dedicated to agriculture and livestock production, being poultry the main activity. In general, producers carry out various productive activities simultaneously. It stands out for its implanted area, the production of forage, fruit trees, fine fruits and leafy vegetables. This work is the first to make numerous aspects of family farming in Santa Cruz visible and presents unpublished information about family farming centers in the province, considering many producers who are not listed as such in other surveys, such as the National Agricultural Census. Having this kind of information is essential for institutions and others social actors in order to monitor their evolution over time and develop policies for their development and strengthening.

Keywords: rural Development; peri-urban production.

\section{INTRODUCCIÓN}

Un referente internacional en agricultura familiar es la Organización de las Naciones Unidas para la Alimentación y la Agricultura (FAO). Esta considera a los agricultores familiares como un grupo grande y muy diverso, definiendo a la agricultura familiar como "una forma de organizar la agricultura, ganadería, silvicultura, pesca, acuicultura y pastoreo, que es administrada y operada por una familia y, sobre todo, que depende preponderantemente del trabajo familiar, tanto de mujeres como hombres. La familia y la granja están vinculados, coevolucionan y combinan funciones económicas, ambientales, sociales y culturales" (FAO, 2014). Como puede verse, la agricultura familiar comprende diversas actividades y se la define como una forma de organización, y en algunos casos como forma de vida, y no como el desarrollo de una actividad puntual de origen agropecuario.

En lo relativo a América Latina Maletta (2011) señala el concepto de unidad económica familiar, que se define como "una finca de tamaño suficiente para proveer al sustento de una familia y que en su funcionamiento no requiriese de mano de obra asalariada, sino que pudiese ser atendida con la fuerza laboral de la propia familia". Asimismo, los países latinoamericanos tienen posturas numerosas acerca de la definición. Por ejemplo, en el caso puntual de los integrantes del Mercado Común del Sur (MERCOSUR) se fijaron directrices para el reconocimiento e identificación de la agricultura familiar. Estas directrices hacen referencia fundamentalmente a la mano de obra utilizada (principalmente familiar), a la responsabilidad de la gestión del espacio productivo y los recursos productivos utilizados, sin considerar otros aspectos relevantes para la caracterización, como el origen y la dimensión de los ingresos familiares o superficie máxima cultivada.

La agricultura familiar abarca a un grupo grande de personas que tienen distintos orígenes y una amplia diversidad cultural, pero que mantienen en común la producción de alimentos como forma de vida. Puede ser por esto que, en Argentina, el Foro Nacional de la Agricultura Familiar (FONAF) defina a la agricultura familiar como una forma de vida y una cuestión cultural, que tiene como principal objetivo la reproducción social de la familia en condiciones dignas, donde la gestión de la unidad productiva y las inversiones en ella realizadas es hecha por individuos que mantienen entre sí lazos de familia, la mayor parte del trabajo es aportada por los miembros de la familia, la propiedad de los medios de producción (aunque no siempre la tierra) pertenece a la familia, y es en su interior que se realiza la transmisión de valores, 
prácticas y experiencias (FONAF, 2006). Esta es una definición que amplía los anteriores conceptos, agregando entre otras cosas la propiedad de los medios de producción, la reproducción social (no solo económica) y la transferencia de conocimiento entre los integrantes de la familia. En este contexto en Argentina, el Registro Nacional de la Agricultura Familiar (ReNAF) y la Secretaria de Agricultura Familiar (SAF) definen al núcleo de agricultura familiar (NAF) basados en los conceptos vertidos por el FONAF y haciendo hincapié en que el origen del trabajo familiar debe estar asociado a la actividad productiva (la familia debe trabajar, gerenciar y administrar el sistema), la compatibilidad de los recursos productivos deben ser compatibles con la capacidad de trabajo de la familia y los ingresos extraprediales de la familia no deben superar los tres sueldos de un peón rural. En este sentido, en el presente trabajo se tomará como unidad de análisis al núcleo de agricultura familiar definido por el ReNAF.

En la provincia de Santa Cruz, existe poca información referida al sector de la agricultura familiar, y sus núcleos (NAF) son predominantemente agrícolas, con condiciones climáticas que influyen de manera significativa en su producción, ya que, comparado con otras zonas productoras del país, el clima templado frío de la mayor parte de la provincia (temperaturas medias entre 0 y $12^{\circ} \mathrm{C}$ ) y la intensidad del viento obligan a los productores a proteger los cultivos y seleccionar mejor las especies a implantar. El nivel de organización de los productores santacruceños nos permite inferir la importancia del sector, ya que la provincia cuenta con más de 20 organizaciones de productores (formales e informales) con capacidad para generar excedentes comercializables principalmente de verduras de hoja, frutas, plantines ornamentales, miel y derivados de la leche. En esta provincia, las producciones de los NAF son diversasy abarcan desde hortalizas y frutas a cultivos forrajeros. Las hortícolas más cultivadas son lechuga (Lactuca sativa), acelga (Beta vulgaris), repollo (Brasica oleracea), papa (Solanum tuberosum), habas (Vicia faba), arvejas (Pisum sativum), ajo (Allium sativum) y una gran variedad de aromáticas como romero (Rosmarinus officinalis), menta (Mentha piperita), tomillo (Thymus vulgaris), melisa (Melissa officinalis) y orégano (Origanum vulgare) (Cepparo, 2013). Estos cultivos se implantan mayormente al aire libre o, en menor medida, en invernáculos construidos con materiales diversos como maderas o hierros y con una cobertura plástica. Los frutales, predominantemente arbustivos, también están muy difundidos, y entre ellos se destacan el cassis (Ribes nigrum), corinto (Ribes rubrum), grosella (Ribes uva crispa) y frambuesa roja (Rubus idaeus) (Birgi, et al. 2019).

Desde el punto de vista de la producción local de alimentos, la agricultura familiar es uno de los sectores de mayor importancia en la región patagónica, no solo por la cantidad y diversidad de los alimentos que produce, sino también por el número de personas involucradas en esta actividad. Sin embargo, sobre este sector no se cuenta con información provincial precisa sobre sus actividades y medios de producción utilizados. Es por esto que cuantificar a los productores y relevar sus condiciones de producción posibilita visibilizarlos, estimar su potencial productivo y obtener información crítica que ayude a colaborar con su desarrollo, no solo para beneficio de los NAF, sino también de la sociedad en su conjunto a través del fortalecimiento de la seguridad y soberanía alimentaria de la provincia. Bajo este criterio, el objetivo del presente trabajo fue caracterizar a la agricultura familiar de la provincia de Santa Cruz, determinando aspectos cualitativos, productivos y condiciones de producción de los núcleos de agricultura familiar. Asimismo, se plantean los siguientes objetivos específicos:

a. Detectar en el relevamiento datos que permitan ser comparados con otras regiones del país y monitoreados en el tiempo. 
b. Establecer criterios de selección que permitan incluir a las producciones familiares predominantes en Santa Cruz, que en otros relevamientos, como el censo agropecuario, quedan excluidos debido al tipo de producción o escala.

\section{Sitio de estudio}

\section{MATERIALES Y MÉTODOS}

El sitio de estudio abarcó un total de 17 localidades de la provincia de Santa Cruz (28 de Noviembre, Caleta Olivia, Cte. Luis Piedra Buena, El Calafate, Fitz Roy, Gobernador Gregores, Jaramillo, Julia Dufour, Los Antiguos, Perito Moreno, Pico Truncado, Puerto Deseado, Puerto Santa Cruz, Puerto San Julián, Río Gallegos y Río Turbio). Dichas localidades constituyen los sitios de mayor población de la provincia.

\section{Metodología de muestreo}

En este trabajo se utilizó como información de base las encuestas relevadas en por el Registro Nacional de la Agricultura Familiar (ReNAF) que fueron provistas por la Subsecretaría de Agricultura Familiar (SAF). Dichas encuestas constituían un total de 23 preguntas, con numerosos sub-ítems, y el detalle de las mismas se puede visualizar en https://www.renaf.magyp.gob.ar/documentos/PLANILLA ReNAF.pdf . Para la realización de este estudio se utilizaron 258 planillas (Tabla 1) de un total de 516 núcleos relevados. Es decir, del total de planillas existentes para Santa Cruz (516), en este trabajo se utilizaron para el análisis de los datos el $50 \%$ de las mismas. Esto se debió fundamentalmente a que al momento de la realización de este estudio, el RENAF llevaba relevadas en Santa Cruz esa cantidad de núcleos de Agricultura Familiar (258). Cada planilla fue completada y relevada en el lugar de emplazamiento del núcleo de agricultura familiar, mediante una entrevista formal al titular e integrantes de cada unos de los NAF de la provincia durante los años 2014-2016. Las mismas constituyen declaraciones juradas de productores familiares de las principales localidades de Santa Cruz y son el resultado de un trabajo de relevamiento conjunto llevado adelante por la SAF, el Instituto Nacional de Tecnología Agropecuaria (INTA) y otras instituciones del sector como el Consejo Agrario Provincial (CAP). Cabe aclarar que para este trabajo se relevaron solo aquellos productores con deseos de participar del relevamiento, por lo que el registro no revistió carácter de obligatorio para ellos.

Tabla 1. Detalle del total de productores familiares relevados en la provincia de Santa Cruz, Argentina, discriminado por localidad y su correspondiente porcentaje con respecto al total de encuestas realizadas.

\section{Localidad Total de NAF relevados Porcentaje}

\begin{tabular}{lcc}
\hline Río Gallegos & 93 & 36,0 \\
\hline Río Turbio & 3 & 1,2 \\
\hline Puerto Santa Cruz & 7 & 2,7 \\
\hline Puerto San Julián & 32 & 12,4 \\
\hline Puerto Deseado & 16 & 6,2 \\
\hline Pico Truncado & 8 & 3,1 \\
\hline Perito Moreno & 5 & 1,9 \\
\hline Los Antiguos & 9 & 3,5 \\
\hline
\end{tabular}




\begin{tabular}{lcc}
\hline Julia Dufour & 9 & 3,5 \\
\hline Jaramillo & 3 & 1,2 \\
\hline Gob. Gregores & 23 & 8,9 \\
\hline Fitz Roy & 5 & 1,9 \\
\hline El Calafate & 6 & 2,3 \\
\hline Cte. Luis Piedra Buena & 9 & 3,5 \\
\hline Caleta Olivia & 17 & 6,6 \\
\hline 28 de Noviembre & 13 & 5,0 \\
\hline \multicolumn{1}{c}{ Total } & $\mathbf{2 5 8}$ & $\mathbf{1 0 0}$ \\
\hline
\end{tabular}

\section{RESULTADOS}

Los NAF en la provincia tienen una superficie media de 5 ha y son administrados por personas que en promedio tienen 47 años. Ciudades como Río Turbio, Fitz Roy, El Calafate y Los Antiguos poseen productores predominantemente adultos, de entre 25 a 45 años de edad. En Caleta Olivia, Cte. Luis Piedra Buena, Jaramillo, Perito Moreno, Puerto Deseado y Río Gallegos, la población se distribuye más homogéneamente predominando los rangos etarios superiores a 25 años por sobre aquellos que poseen una edad menor. El dato más destacable es que solo un $5 \%$ de todos los titulares de NAF poseen menos de 25 años, siendo uno de los rangos etarios de menor participación en la mayoría de las localidades de la provincia (Figura $1)$.

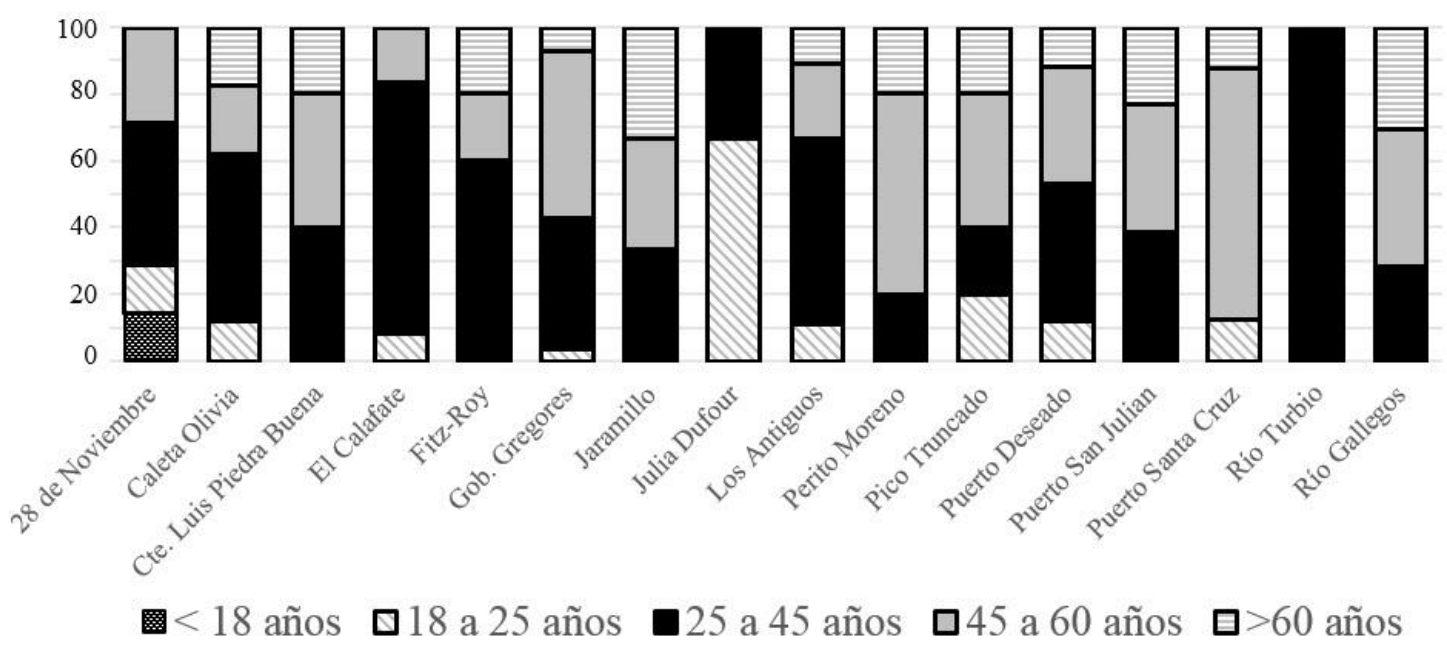

Figura 1.Rangos de edad de los titulares de Núcleos de Agricultura Familiar (NAF) por localidades de la provincia de Santa Cruz.

\section{Principales producciones del NAF}

La localidad con mayor superficie en producción dentro del sector de la agricultura familiar es El Calafate, siguiendo en orden de importancia Los Antiguos, Gobernador Gregores, Julia Dufour, Río Gallegos y Puerto Deseado (Figura 2). 


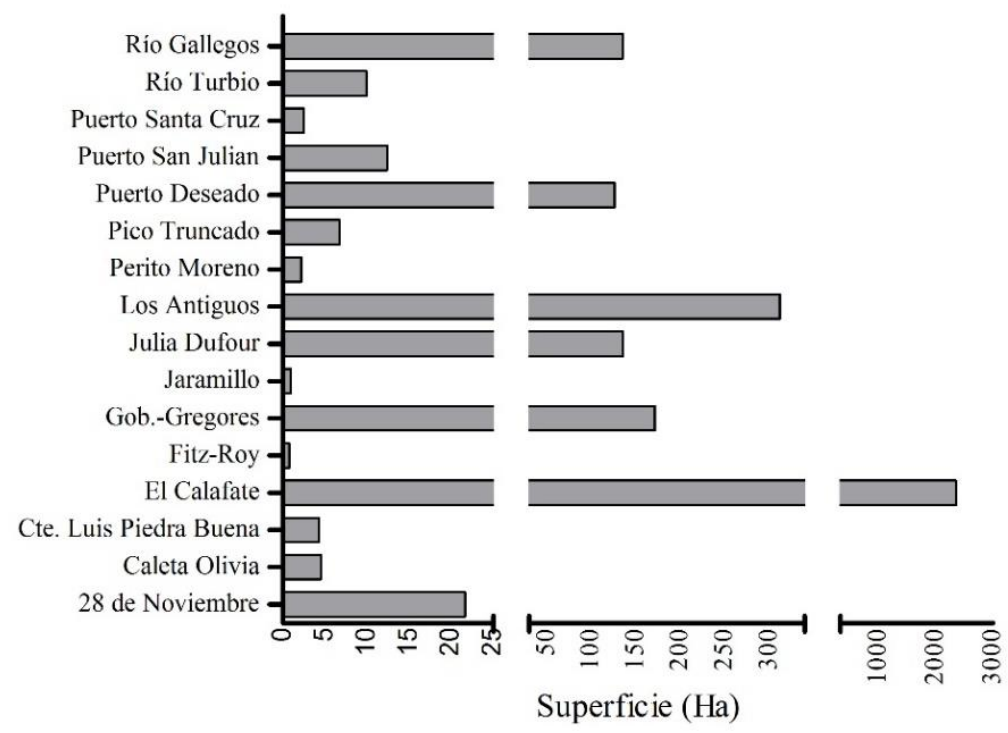

Figura 2.Superficies con producción familiar de las principales localidades productivas de la provincia de Santa Cruz.

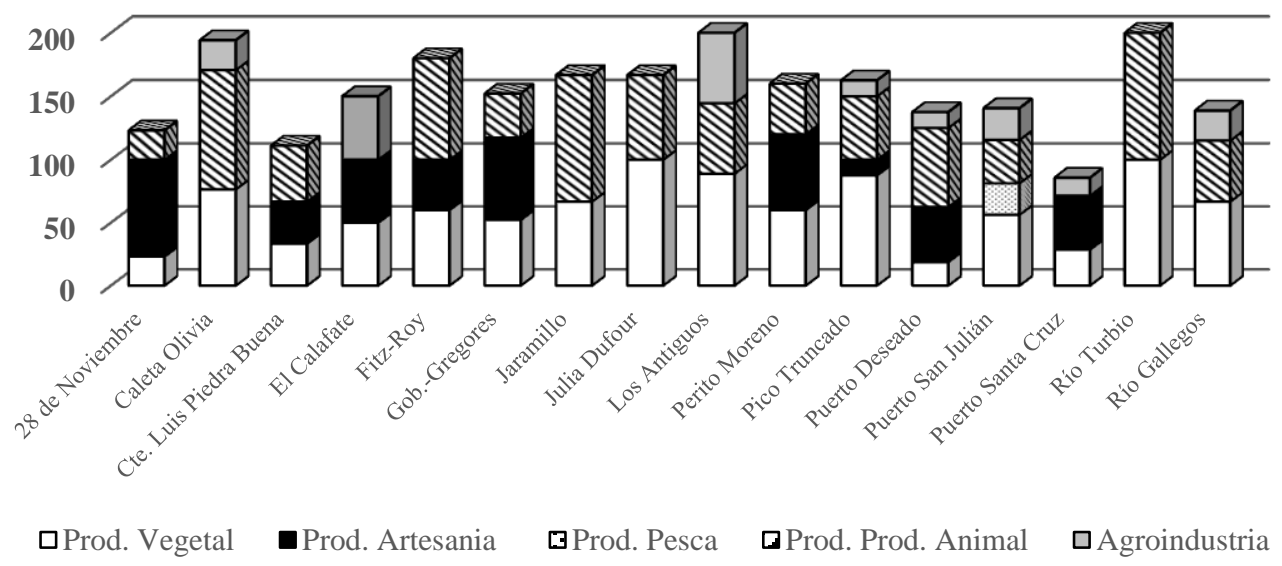

Figura 3.Detalle de la participación porcentual (\%, eje Y) de las principales actividades de producción familiar diferenciadas por localidad para la provincia de Santa Cruz.

Discriminando las principales actividades por localidad, en la Figura 3 se observa la predominancia de la producción vegetal y animal por sobre las demás actividades productivas como la pesca, la agroindustria o la producción de artesanías. Esto se da sobre todo en localidades como en Río Turbio, Río Gallegos, Julia Dufour, Jaramillo y Caleta Olivia. Asimismo es destacable tanto la participación de la producción de artesanías en la provincia, como también la relativa importancia que presenta la actividad pesquera en la producción familiar de Puerto San Julián.

En general, los productores realizan diversas actividades productivas en simultáneo (Figura 3). Las principales actividades realizadas están relacionadas con la producción vegetal (más de $60 \%$ de los productores las realizan), y dentro de ésta, la actividad predominante desde el punto de vista del área bajo cultivo es la producción de forrajes, que tiene 158 hectáreas, seguida de la producción de frutales varios con 46,5 ha y las frutas finas con 46 hectáreas (Figura 4). Otra actividad productiva a destacar por su superficie es la de hortícolas de hoja, 
que suma 33,5 hectáreas, y que como se verá más adelante, cobran relevancia por sus volúmenes de producción.

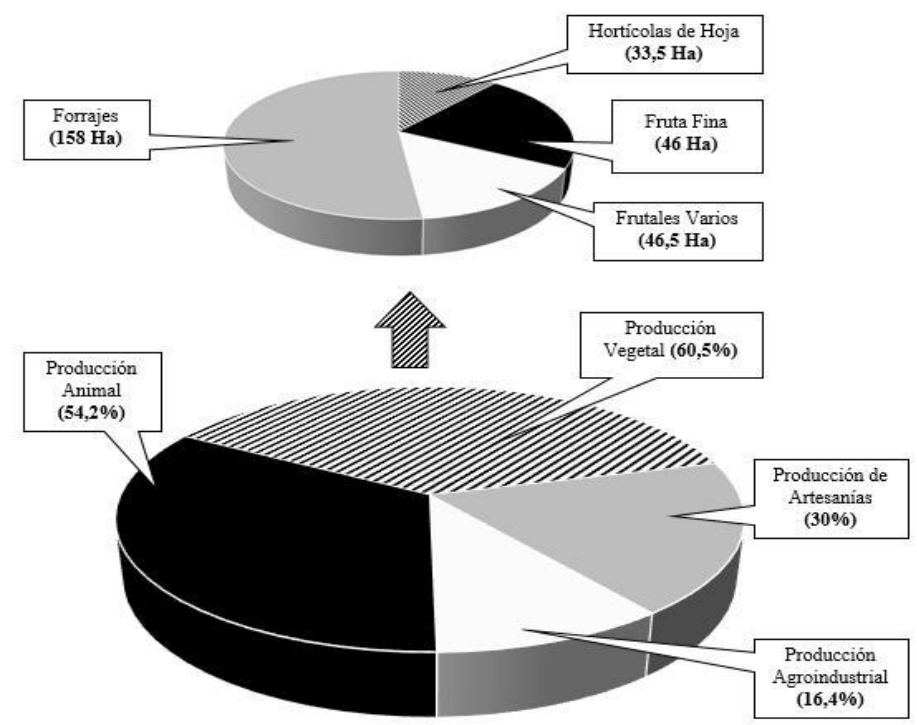

Figura 4.Actividades productivas familiares de mayor difusión y principales producciones vegetales (en cuanto a superficie) de los agricultores familiares de la provincia de Santa Cruz.

En cuanto a la producción de artesanías, se observó que solo el 18,3\% de los artesanos produce su propia materia prima, es decir que en general las artesanías santacruceñas no son elaboradas con materias primas generadas en el seno del NAF. Un ejemplo a citar podría ser el de las localidades que se encuentran próximas a la cordillera de los Andes, lugar en donde se puede encontrar bosques nativos con maderas de excelente calidad que no estarían siendo aprovechadas en la producción de productos de origen maderero. A este ejemplo se le suma el de la producción de lanas e hilados que reviste gran importancia dentro del rubro (Figura 5) y en donde tampoco se produce la materia prima dentro del NAF, aunque las condiciones generales de la provincia así lo permitan.

En la actividad agroindustrial se evidencia un mayor concatenamiento con otras actividades debido al hecho de que el $53 \%$ de los productores dedicados a la actividad agroindustrial llevan adelante también una actividad relacionada a la producción vegetal o animal con la que generan materias primas que luego son usadas en la producción de derivados agroindustriales. De ahí que el $44 \%$ de los productos derivados de la agroindustria está directamente relacionado a la producción vegetal, ya que se trata fundamentalmente de dulces, mermeladas, hortalizas fraccionadas y bebidas. En este sentido, la actividad agroindustrial se comporta como un complemento de otras actividades, principalmente agrícolas, como una forma de agregar valor a la producción. 


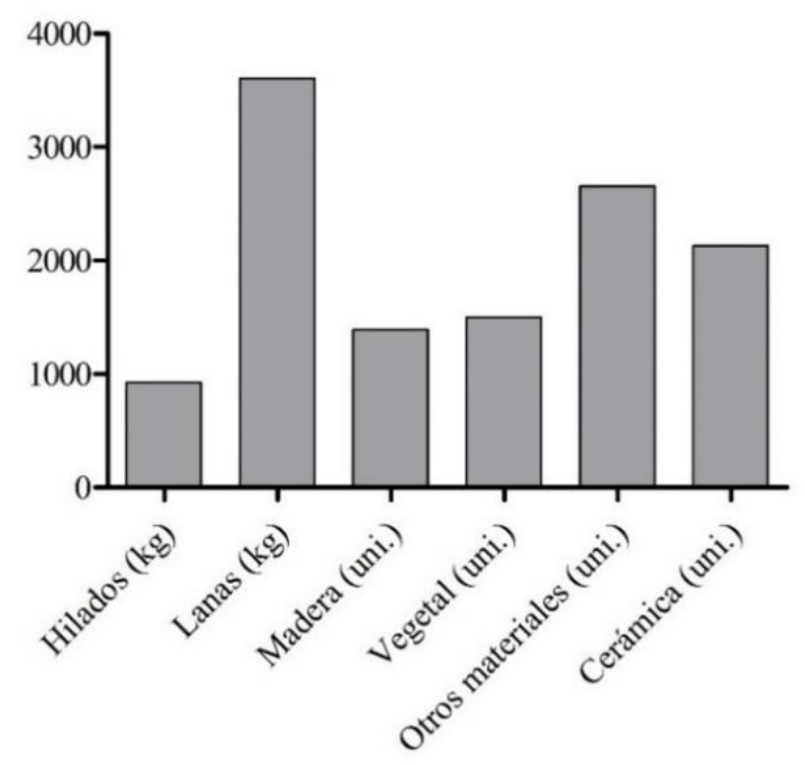

Figura 5. Detalle de los distintos tipos de artesanías de origen familiar (eje X) y su volumen de producción en kilogramos y/o unidades producidas (eje Y) para la provincia de Santa Cruz.

\section{Producción Vegetal}

La información disponible sobre las condiciones de producción del NAF agrícola incluye datos de infraestructura, insumos de producción, métodos de control de plagas y uso de la rotación de los cultivos. Entre los resultados obtenidos se destaca que Santa Cruz cuenta con un $89 \%$ de productores con disponibilidad de agua para producciones agrícolas. En cuanto al método de distribución del agua la información indica que casi el $60 \%$ de los productores riega sus cultivos de forma manual y solo el $20 \%$ utiliza sistemas más complejos como el riego por goteo o en menor medida (10\%) aspersión.

Con respecto a la infraestructura, el $33 \%$ de los productores consultados poseen invernaderos en sus chacras productivas. Si de estos se selecciona solo a quienes producen especies hortícolas, el porcentaje de productores que disponen de esta tecnología se incrementa a $46 \%$. Otra infraestructura para la producción que reviste importancia es el galpón o tinglado para el almacenamiento de la producción, los insumos o los equipos necesarios para producir. En este sentido encontramos que un $30 \%$ de los productores tiene un galpón instalado y que casi un $4 \%$ posee un tinglado. Este último caso tiene un porcentaje menor debido posiblemente a que el tipo de infraestructura no está adaptada a la intensidad de los vientos reinantes en Patagonia sur.

Entre los bienes muebles que debería poseer el productor familiar en Santa Cruz se encuentra el tractor. En este caso el porcentaje de productores que lo posee es extremadamente bajo, ya que el equipo se encuentra disponible en menos del $2 \%$ de los productores familiares de la provincia.

De los insumos de producción que se necesitan en la agricultura familiar el que probablemente posea mayor criticidad es la semilla, y en este sentido el $35 \%$ de los productores familiares santacruceños produce su propia semilla, pero además el $66 \%$ de estos declaran recibir semillas subsidiada por un organismo del estado.

En cuanto al fertilizante, más del $74 \%$ de los productores utiliza un fertilizante orgánico de producción propia y solo un $13 \%$ aplica fertilizantes de síntesis química que compra en el mercado. Una lógica que se repite con el uso de insecticidas, ya que solo el $17 \%$ aplica 
productos de síntesis química, mientras que el $31 \%$ utiliza métodos no químicos para el control de las plagas.

Si bien en la actualidad Santa Cruz no se autoabastece de productos frutihortícolas, en la provincia se producen anualmente cerca de 220 toneladas de estos productos de origen familiar. Los volúmenes de producción para las frutas finas, por ejemplo, superan las 31 toneladas anuales distribuyéndose en un poco más de 46 ha de cultivo. La situación de las frutas finas es similar a la encontrada en la producción de frutales varios ya que en una superficie de 46,5 ha se produce un volúmenes de 34 toneladas anuales.

Si se pone énfasis en la cantidad de producto obtenido, la producción de hortícolas de hoja es la principal actividad agrícola de la provincia, logrando anualmente 69,3 toneladas de producto (Figura 6) con un poco más de tres hectáreas de producción. Otra actividad a destacar (en cuanto a volumen) es la producción de legumbres, bulbos y tubérculos, ya que ésta supera las 37 toneladas anuales (Figura 6) y representa el 17\% del total de todos los productos que se obtienen anualmente en la provincia. Por último, cabe mencionar a la huerta de autoconsumo, con una producción que supera los 5500 kilos y que representa en muchos casos una producción complementaria a las demás actividades.

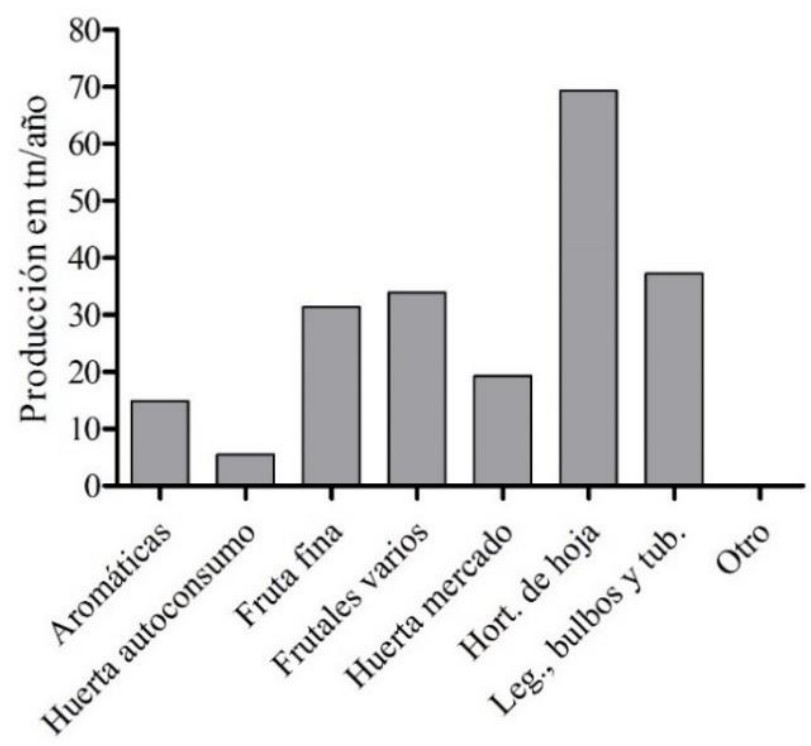

Figura 6. Rendimiento (toneladas por año) de las distintas producciones agrícolas familiares de la provincia de Santa Cruz.

\section{Producción Animal}

Del análisis de las encuestas surge que la producción pecuaria es realizada por más de la mitad de los productores $(54,2 \%)$. De las condiciones de producción para estas actividades se destaca el alto porcentaje de productores que tiene disponibilidad de agua para sus animales (89\%) y además la disponibilidad de infraestructura de producción (galpones, jaulas, gallineros) en el seno del NAF que alcanza el $83 \%$ de productores. Este último dato contrasta mucho con la disponibilidad de herramientas y equipos (ordeñadoras, balanzas, esquiladoras) del NAF pecuario, ya que para llevar adelante la actividad solo el $7 \%$ posee lo necesario para producir.

En cuanto al manejo, solo el $25 \%$ de los productores realiza aplicaciones químicas para el control de enfermedades, mientras que el $22 \%$ lo hace a través de medios no químicos o biológicos no especificados en el instrumento de relevamiento. Además hay que destacar que 
en los NAF pecuarios el principal método de mejoramiento genético es el cruzamiento con un $24 \%$ del total, seguido de la selección con un $22 \%$ y una minoría del $3 \%$ que practica inseminación artificial en sus animales. Por último es destacable que más de la mitad de los NAF pecuarios $(56 \%)$ disponga de medios de movilidad para realizar las tareas necesarias para llevar adelante la actividad. Esto toma relevancia ya que por normativas vigentes en la mayoría de las localidades establecen que los lugares de producción deben encontrarse fuera del ámbito urbano.

Dentro de las producciones pecuarias podemos encontrar desde ganado porcino y ovino hasta producción avícola y conejos (Oryctolagus cuniculus). En este sentido, la producción más importante en cuanto al número de productores es la avícola, ya que la llevan adelante un 57\% de los NAF, seguida por la producción porcina, con un $27 \%$ del total de núcleos relevados. Siguiendo con el caso de la producción avícola hay que destacar que más del $80 \%$ de los productores se dedica a la producción de líneas livianas (ponedoras) y el resto de ellos a las líneas pesadas (pollos camperos, parrilleros, entre otras) o especies diversas como la codorniz (Coturnix coturnix), pavo (Meleagris gallopavo), ganso (Anser anser domesticus) o pato doméstico (Anas platyrhynchos domesticus). En cuanto a los volúmenes de producción, cobra notoriedad la producción de huevos frescos por sobre la producción de carne (de aves), ya que se producen unas 58.580 docenas anuales con un promedio aproximado de 732 docenas por productor, comparado con 2.300 pollos faenados anualmente que solo promedian $76 \mathrm{~kg}$ por productor familiar. En la provincia además existen otras actividades avícolas como las producciones alternativas de aves (pavos, patos, gansos) que alcanzan unos 1.000 ejemplares anualmente o la producción de conejos con más de 2.300 animales al año. Estas se desarrollan generalmente de manera complementaria a las producciones principales de la chacra y por lo general son orientadas al autoconsumo familiar o a la venta a eventuales compradores. Por otro lado entre las producciones pecuarias se encuentra la producción porcina con volúmenes anuales que rondan los 2.100 lechones (animales de entre 8 y $12 \mathrm{~kg}$ faenado) y unos 10.841 kilos de carne anuales (obtenida de animales adultos de más de $100 \mathrm{~kg}$ ). Aunque las unidades de producción no son comparables, con estos valores se puede inferir que desde el punto de vista de los volúmenes de producción, la porcicultura y la avicultura son las principales actividades pecuarias de la provincia.

\section{Artesanías y Agroindustria}

Otras actividades de relevancia que se desprenden del relevamiento son las artesanías y la agroindustria. En el caso de las artesanías se puede evidenciar productores más independientes respecto de otras actividades, ya que del total de productores que se declaran artesanos más del $60 \%$ no lleva adelante otra actividad productiva dentro del NAF. Asimismo, las artesanías derivadas de la actividad ovina cobran particular relevancia, esto puede deberse a un aspecto cultural, la disponibilidad de la materia prima o la combinación de ambas que interactúan para hacer de esta actividad una de las más importantes dentro de este rubro. Por otro lado hay que destacar la participación de los productos de origen vegetal y madereros, que junto a la producción de cerámicas y otros materiales completan la clasificación de los productos producidos regionalmente.

En cuanto a los volúmenes de producción de la agroindustria se pueden destacar productos como las hortalizas procesadas, con unas 24 toneladas anuales, las conservas, que superan los $4.000 \mathrm{~kg}$ o las mermeladas y jaleas con más de $3.000 \mathrm{~kg}$ anuales de producción. Asimismo en la provincia se producen otros productos agroindustriales como ahumados y aderezos que alcanzan los $347 \mathrm{~kg}$ al año o las bebidas con un volumen de producción anual de más de 3.200 litros. 


\section{El trabajo, la residencia y los servicios del NAF}

De la información obtenida en el relevamiento, se observa que cerca del $63 \%$ de los integrantes del NAF trabajan en las actividades de producción dentro del núcleo y la fuerza laboral dentro de este es homogénea en cuanto al género. También puede observarse que entre el 62 y el $70 \%$ de los hombres y mujeres cuentan con un trabajo fuera del NAF (Figura 7), siendo el $31 \%$ de estos jubilados. Por los ingresos extraprediales que percibe el NAF se puede inferir que existen dificultades en la producción como único sustento, ya que solo un 17\% del total de productores no percibe ningún tipo de ingresos extraprediales en la provincia. Asimismo existen distintas retribuciones que engrosan los ingresos extraprediales del NAF, las cuales se pueden ver en la Figura 7 en donde se destacan la Asignación Universal por Hijo (AUH), otros tipos de asistencias sociales y el alquiler de terrenos por parte del núcleo.

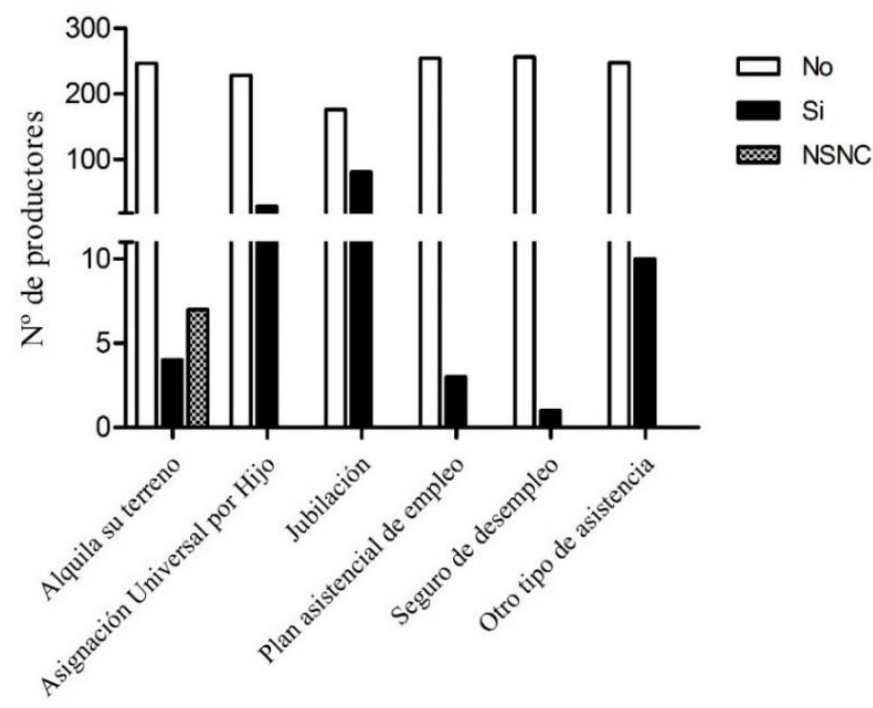

Figura 7. Número de productores familiares que reciben ingresos extraprediales y contribuciones sociales clasificados en función del tipo de retribución. (NSNC: no sabe, no contesta).

En cuanto a la residencia en donde habita el núcleo y los servicios a los que éste puede acceder se puede decir que el $86 \%$ de los NAF tienen suministro de energía eléctrica y el $44 \%$ de todos ellos accede al servicio de cloacas. El suministro de agua de red en los NAF alcanza al $72 \%$ de las viviendas, mientras que el resto se garantiza el suministro principalmente mediante pozo (11\%) o perforación ( $8 \%)$. Además existen otros medios de suministro de agua como la lluvia, canales, arroyos o ríos que se utilizan como provisión complementaria.

Por otro lado titulares de NAF declaran que el $83 \%$ de los hogares tiene una cocina instalada y que en el $60 \%$ de los casos la vivienda cuenta con suministro de gas natural. En el caso de no tener al gas natural como principal combustible, el núcleo familiar usa en un alto porcentaje garrafas sin subsidios del estado, situación que representa el $25 \%$ de los casos, solo un $15 \%$ reciben ayuda del gobierno. Por último, un poco más del $6 \%$ de los NAF deben usar leña (u otros combustibles) para su aprovisionamiento de energía.

\section{Condiciones de producción y comercialización}

Las condiciones climáticas de Santa Cruz impactan de manera negativa en el sistema productivo familiar ocasionando eventualmente mermas en los rendimientos de algunas especies o incluso impidiendo su producción. Esta situación puede verse más claramente en aquellos productores que no implementan medidas de protección para sus cultivos o que 
instalan estructuras inadecuadas o deficientes para el tipo de producción que realizan. En este sentido hay que destacar que sin importar la actividad que desarrolle el productor familiar, el $75 \%$ de ellos declara que su producción corre riesgo debido a las condiciones climáticas o a factores biológicos (Figura 8). Asimismo más de la mitad (58\%) de los productores que declaran estos riesgos en sus producciones informan que no toman ninguna medida de prevención para evitarlos, y este escenario se agrava aún más si se piensa en atenuar el impacto negativo por medio de la contratación de algún seguro, ya que según el relevamiento el porcentaje de productores que contrató un seguro solo llega al $2 \%$, destacando además, que estos productores no están relacionados directamente con las principales producciones de la provincia, sino con la producción de artesanías.
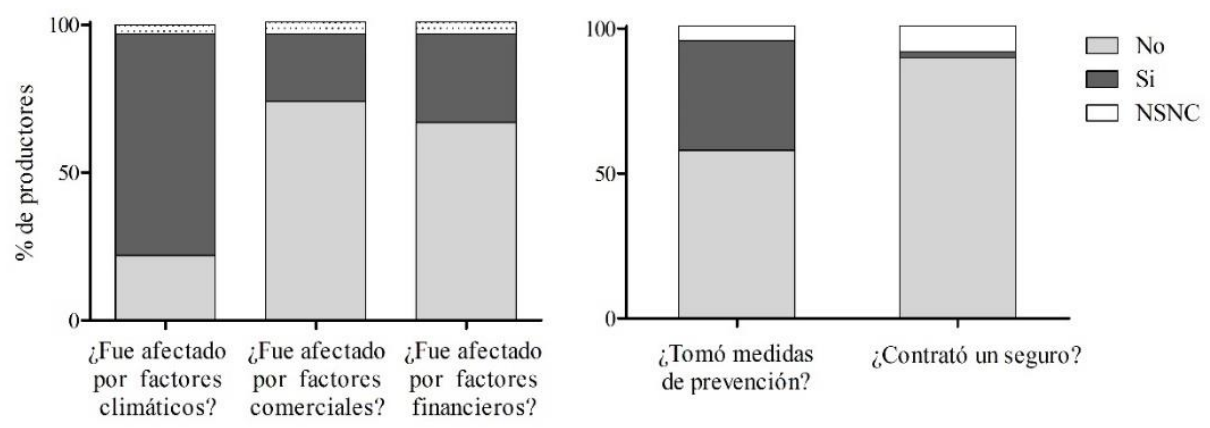

Figura 8. Factores que afectan la producción de los productores familiares de Santa Cruz medidas tomadas para revertir las consecuencias. NSNC: No sabe no contesta.

Por último en la producción de los NAF los factores comerciales y financieros afectan solo a un 20 o $30 \%$ del total de productores, una cifra alentadora que denota entre otras cosas el interés local por los productos generados.

\section{CONCLUSIONES Y PROPUESTAS}

Hasta la década del 60 en Santa Cruz comprar vegetales en las chacras era la única forma de consumir frutas y verduras frescas en la región, ya que por ese entonces no se disponía de infraestructura vial adecuada para la circulación fluida de los camiones necesarios para el aprovisionamiento de alimentos producidos en otras provincias. Esos años fueron muy fructíferos para los productores familiares y propiciaron su rápido crecimiento, favoreciendo además la incorporación de nuevas infraestructuras de producción y mejoras, como la instalación de pozos de agua y luz eléctrica.

Con la llegada del pavimento a la Patagonia se abrieron nuevas vías de comercialización entre las provincias productoras de alimentos y Santa Cruz, con lo cual comenzaron a arribar camiones de transporte y empresas prestadoras de este servicio. Esta situación incrementó la afluencia de nuevos insumos y alimentos a la provincia, produciendo como consecuencia la proliferación de comercios dedicados a la reventa de frutas y verduras que con el tiempo fueron desplazando a los productores familiares del mercado que no supieron adaptarse a esta situación.

La agricultura familiar es un sector complejo que presenta dificultades al momento de ser cuantificado. Los diversos criterios de clasificación y la escasa información disponibles a nivel provincial dificultan su medición. Esto queda evidenciado al compilar la información 
disponible en donde se observa discrepancias en los criterios de relevamiento y la unidad geográfica mínima analizada. En este sentido, y a modo de resumen, la Tabla 2 sintetiza la cantidad de productores familiares por localidad (o Departamento) según distintos actores gubernamentales que realizaron relevamientos en la provincia.

Tabla 2. Total de productores familiares discriminados por localidad (o Departamento) según las principales instituciones vinculadas al sector de la agricultura familiar.

\begin{tabular}{|c|c|c|c|}
\hline Localidad & $\begin{array}{l}\text { INDEC (EAPs) } \\
(2002)^{*}\end{array}$ & $\begin{array}{l}\text { ProHuerta } \\
(2011)^{* *}\end{array}$ & $\begin{array}{c}\text { SAF y presente trabajo } \\
(2017)\end{array}$ \\
\hline 1-Río Gallegos & - & 1.060 & 114 \\
\hline 2-Río Turbio & - & 68 & 16 \\
\hline 3-Puerto Santa Cruz & - & 47 & 16 \\
\hline 4-Puerto San Julián & - & 106 & 34 \\
\hline 5-Puerto Deseado & - & 75 & 22 \\
\hline 6-Pico Truncado & - & 140 & 26 \\
\hline 7-Perito Moreno & - & 92 & 16 \\
\hline 8-Los Antiguos & - & 142 & 26 \\
\hline 9-Julia Dufour & - & - & 5 \\
\hline 10-Jaramillo & - & - & 4 \\
\hline 11-Gob. Gregores & - & 109 & 37 \\
\hline 12-Fitz Roy & - & - & 5 \\
\hline 13-El Calafate & - & 226 & 22 \\
\hline 14- Cte. Luis Piedra Buena & - & 33 & 45 \\
\hline 15-Caleta Olivia & - & 529 & 110 \\
\hline 16-28 de Noviembre & - & 24 & 18 \\
\hline Dpto. Corpen Aike $(3+14)$ & 83 & - & - \\
\hline Dpto. Deseado $(5+6+10+12+15)$ & 314 & - & - \\
\hline Dpto. Güer Aike $(1+2+9+16)$ & 110 & - & - \\
\hline Dpto. Lago Argentino (13) & 111 & - & - \\
\hline Dpto. Lago Buenos Aires $(7+8)$ & 146 & - & - \\
\hline Dpto. Magallanes (4) & 76 & - & - \\
\hline Dpto. Rio Chico (11) & 107 & - & - \\
\hline Total & 947 & 2.651 & 516 \\
\hline
\end{tabular}

*Resultados discriminados por Departamento.

**Número de kits de semillas entregados según el informe anual de campaña 2.011-2.012 de la coordinación provincial de ProHuerta Santa Cruz.

Una de las principales problemáticas del sector es el problema de la edad de los productores que en la provincia alcanza un promedio de 47 años. En este sentido, cabe aclarar que si bien existen factores que claramente modifican la pirámide etaria de la población como la natalidad o la mortalidad, la principal causa del envejecimiento de la población agrícola familiar de Santa Cruz está relacionada con la migración, sobre todo en la población más joven que busca en la ciudad oportunidades de crecimiento que la agricultura familiar no ofrece. Según la Organización Mundial de la Salud (OMS) (2002) las personas de 60 años son consideradas de edad avanzada o ancianos. En este sentido cerca del $20 \%$ población de 
agricultores familiares de la provincia entran en este rango etario, y un $37 \%$ de ellos tienen edades que van desde 45 a los 60 años. Esto constituye un dato relevante al momento de plantear estrategias destinadas al sector y colabora además en comprender cuál es la capacidad de trabajo actual de los productores en términos generales. En concordancia con estos datos, el Proyecto para el Desarrollo de Pequeños Productores (PROINDER) determina un descenso de población rural santacruceña del 45\% entre el año 1991 y el 2001 (Biaggi, Cristina, Canevari Cecilia \& Alberto Tasso, 2007). En este sentido se considera prioritario crear herramientas específicas que permitan promover la generación de nuevos productores familiares y la capacitación de mano de obra familiar para lograr una transformación tecnológica de la agricultura tradicional. Esto significa que las reformas educativas deberán formar investigadores que comprendan mejor las necesidades de los campesinos (Brundtland et al., 1987) e implica generar "usinas" de nuevos productores que puedan incorporarse a la población ya existente. Para ello una alternativa posible podría ser la incorporación de las asociaciones de productores como plataformas de capitalización para las personas interesadas en iniciarse en la actividad. En este esquema las asociaciones serían las receptoras de los subsidios o créditos blandos y los administrarían para la compra de insumos, herramientas y equipos para el trabajo cooperativo, además priorizarían la construcción de infraestructuras (principalmente invernáculos) que permitan la prestación de servicios como la multiplicación de plantines con un mercado real dentro del mismo sector. Esto permitiría retroalimentar a los productores familiares con servicios e insumos críticos que ellos mismos producirían y que hoy compran en otras provincias del norte del país.

Por otro lado la asociación podría cobrar un canon por el uso de las instalaciones y equipos que se podría destinar a la conformación de un fondo rotatorio que se utilizaría para generar un stock de los insumos críticos de las principales producciones (muchos escasos en la zona) o para financiar a los productores en la iniciación de sus propias chacras productivas. Además la metodología planteada podría complementarse con herramientas ya propuestas para el sector como el Programa ProHuerta, Cambio Rural o la participación de la SAF, que además de acompañar con algunos de los insumos críticos, financiamiento y monotributo social agropecuario, aportarían asesoramiento técnico y acompañamiento en terreno para el nuevo productor y la asociación.

\section{Principales producciones y condiciones de producción}

De los resultados obtenidos en el relevamiento se destacó la participación de algunos cultivos como los forrajeros, la fruta fina o la producción de verduras de hoja. En este sentido es notable la participación local de las especies forrajeras, ya que a nivel nacional solo constituyen un $12 \%$ de las producciones realizadas en los NAF y en Patagonia alcanzan un $22 \%$ (RENAF, 2014). Además se puede agregar que el resto de los principales cultivos realizados en la provincia tampoco constituyen actividades principales en el país o Patagonia, ubicándose en el primer caso (nivel país) por debajo de las tres actividades más realizadas.

Un aspecto que se debería considerar para potenciar estas producciones es el modo en que se usa el agua de riego, ya que aunque el $89 \%$ de los productores la tiene disponible para sus cultivos, solo el $20 \%$ utiliza sistemas adaptados a la región como el riego por goteo. Esta situación constituye un obstáculo a la producción, si se consideran las condiciones climáticas de la provincia, ya que por lo general el viento predominante incrementa la tasa de evaporación del agua en el suelo, aumentando por ende la frecuencia de riego necesaria para mantener el cultivo. Además el riego manual (principal método de riego en la provincia) demanda una mayor mano de obra y tiempos operativos. La implementación de riegos más tecnificados permitiría no solo un incremento en la eficiencia de uso del recurso, sino también una disminución en el costo de mano de obra y mejoras en el estado sanitario de las plantas, 
ya que con el riego manual se fomenta, por ejemplo, la proliferación de enfermedades a nivel de cuello en cultivos susceptibles como los de fruta fina u otros cultivos como el tomate o el pimiento. Sin embargo, la utilización de otros sistemas como el riego por aspersión (instalado en el $10 \%$ de los NAF) presenta también dificultades de uso, ya que la entrega del agua con este método es muy heterogénea debido a la acción del viento. Por lo que se concluye que en base a los cultivos predominantes en la región y las condiciones climáticas de la zona la opción más adaptada a las producciones locales es el riego por goteo, que además se puede automatizar con una relativa baja inversión, es más eficiente en el uso del agua (Leonardo, 2015) y homogéneo en su entrega.

En cuanto a la infraestructura y los equipos utilizados en la agricultura familiar de Santa Cruz se destacan por un lado el alto porcentaje de productores con invernaderos en producción (33\%) y la escasa disponibilidad de maquinaria (principalmente tractores) que limita el potencial productivo de los NAF, ya que esta herramienta se torna imprescindible para el laboreo de los suelos, que por lo general tienden a ser pesados y difíciles de labrar.

Del relevamiento surge además que por lo general las producciones en la provincia son mixtas con sistemas diversificados que producen manufacturas de naturaleza agrícola-pecuarias. Esta realidad supone que las instituciones abocadas al fortalecimiento del sector deban tener una visión amplia del sistema (y más compleja), ya que no se trata solo de familias realizando una huerta familiar o criando algunas gallinas cómo se las simplifica muchas veces, sino de productores altamente diversificados con un gran potencial productivo que muchas veces no cuentan con las herramientas necesarias para expresar esas potencialidades. Al respecto, Brundtland et al. (1987) expresó "la investigación debe acordar prioridad especial a las necesidades diversas de la agricultura mixta, típica de los cultivos de subsistencia. Se deben movilizar los sistemas de extensión y de abastecimiento de insumos, a fin de que alcancen a los agricultores". Asimismo una visión parcial o sesgada de la realidad productiva de los NAF puede llevar a plantear el uso de paquetes tecnológicos o políticas de fomento que no se adaptan a la realidad de los núcleos y que aunque aporten recursos al sector, no implican necesariamente el desarrollo de estos. En este contexto, la búsqueda de nuevos métodos de producción y alternativas productivas adaptadas al sector (como la producción de frutas finas bajo cubierta) es de vital importancia para su fortalecimiento y deberían constituir líneas prioritarias de trabajo en instituciones como el INTA o la SAF.

Un aspecto a destacar relacionado con las condiciones de producción son las legislaciones vigentes para la venta de alimentos, particularmente aquellas que hacen hincapié en las condiciones de producción y comercialización. Localmente no se hace diferencia desde lo formal entre las grandes producciones agroindustriales y las producciones familiares, exigiendo a los NAF instalaciones similares a las usadas por la industria para la elaboración de sus productos. En este sentido es necesario un acompañamiento más estratégico por parte de los organismos de control y las instituciones relacionadas a estas producciones, sobre todo en aspectos centrales como la instalación de frigoríficos municipales o cooperativas de trabajo para que los productores puedan acceder a una faena adecuada de sus animales y la elaboración de sus conservas en lugares habilitados para tal fin. Asimismo es necesaria la colaboración técnica de los municipios para llevar adelante los análisis de calidad sobre la mercadería, sobre todo aquellos análisis destinados a detectar triquinosis en porcinos o las necropsias en aves para permitir el correcto diagnóstico de enfermedades potencialmente peligrosas para la salud. 


\section{El trabajo, la residencia y los ingresos extraprediales en el NAF}

En cuanto al trabajo que se lleva adelante en el NAF se relevó significativa participación de sus integrantes en las actividades productivas del núcleo, con homogeneidad de género. Al respecto de los aportes realizados por las mujeres en el ámbito rural es probable que en la actualidad existe una escasa valoración y visibilización de este trabajo, e inclusive las mismas mujeres no advierten la cantidad de horas que trabajan al día y como esto limita su participación en actividades comunitarias y sociales (Biaggi, Cristina, Canevari Cecilia \& Alberto Tasso, 2007). Asimismo y como explican estos autores "cuando las mujeres reemplazan a los varones en las tareas prediales, no siempre implica que pueden tomar decisiones sobre los recursos de la finca y eso debilita el proceso productivo". Según la FAO (2011) "los datos relativos a África, Asia y América Latina muestran sistemáticamente que las familias salen beneficiadas cuando las mujeres mejoran su estatus y su poder aumenta en el hogar", además en el mismo informe se agrega que "cuando las mujeres tienen más influencia sobre las decisiones económicas, sus familias destinan más ingresos a la alimentación, la salud, la educación, el vestido y la nutrición de los niños”. Es por esto que la organización de la fuerza de trabajo de los productores familiares es clave valorizar el rol de la mujer, y que además se debería propender a una participación más activa de ésta en la gestión de los NAF y los grupos cooperativos o asociativos que colaboren en el desarrollo de los productores familiares en la región.

La homogeneidad de género es una información de importancia superlativa por su diferenciación mundial y nacional en este aspecto, ya que desde el punto de vista internacional según la FAO (2011) las mujeres representan en promedio el $43 \%$ de la fuerza laboral agrícola de los países en desarrollo y su proporción particularmente en América Latina se aproxima al $20 \%$. Asimismo hay que destacar que según Arizpe (1986) en general la migración del ámbito rural en países como México o el resto de América Latina han sido preponderantemente femenina y destacó que la proporción de ésta fue de 100 mujeres por cada 85 hombres.

A nivel local según el informe "Mujeres que Trabajan la Tierra" del Proyecto para el Desarrollo de Pequeños Productores (PROINDER) la migración femenina fue significativamente mayor que la de los varones en la provincia de Santa Cruz (Biaggi, Cristina, Canevari Cecilia \& Alberto Tasso, 2007). El informe agrega además que en el ámbito rural existe una predominancia del género masculino que se ve reflejada en todo el territorio nacional, remarcando que esta tendencia es más evidente en las provincias patagónicas, ya que por ejemplo la ruralidad alcanza una proporción de $70 \%$ de hombres y $30 \%$ de mujeres para los habitantes rurales de la provincia de Santa Cruz.

Otro aspecto de interés que requiere de una reflexión es el requisito de los ingresos monetarios extraprediales que perciben los integrantes del ReNAF. En este sentido el Registro deja establecido como límite máximo tres sueldos de un peón rural para ser considerado productor familiar. Este límite resulta bajo para algunas zonas alejadas, como Santa Cruz o Tierra del Fuego, en donde la distancias a los centros productivos son amplias, tanto para el abastecimiento de alimentos o como de la mayoría de los insumos críticos necesarios para producir, como por ejemplo fertilizantes, agroquímicos o material de propagación. Es por esto los costos de reproducción de los NAF no son los mismos que los de otras regiones del país, por lo que se debería considerar la implementación de nuevos límites de ingresos extraprediales que consideren la zona en donde el NAF produce, el costo de vida de los integrantes del núcleo y el grado de protección que requieren los cultivos para su implantación. De esta forma se podrá incluir a algunos productores que quedan afuera del registro por no cumplimentar con los máximos establecidos. 
En cuanto a los servicios disponibles en la residencia del NAF la información del relevamiento indica que los núcleos santacruceños poseen comparativamente una mayor disponibilidad de servicios comparados con otras regiones del país. Por ejemplo, el $72 \%$ de los núcleos de la provincia poseen suministro de agua de red, comparado con un $19 \%$ que se registra en el resto de Patagonia o 34\% que se informa a nivel nacional (RENAF, 2014). Además en Santa Cruz el $86 \%$ de los NAF tienen red eléctrica y en el resto del país este número es de $63 \%$, disminuyendo a $47 \%$ si solo se considera a Patagonia. La alta disponibilidad de estas prestaciones dentro de los núcleos de agricultura familiar posibilita el arraigo de las familias al lugar, facilita la instalación del hogar en el lugar de producción y simplifica las tareas operativas, promoviendo además el desarrollo de actividades como la cría de animales que requieren una mayor atención.

En conclusión, el presente trabajo no solo brinda información interesante acerca de los productores familiares de la provincia de Santa Cruz, sino que también permite visibilizar al sector y comparar los aspectos socioproductivos de sus NAF, con otros relevados en el resto del país. La factibilidad de uso de esta información abarca no solo instituciones públicas y privadas nacionales y provinciales, sino también a los productores familiares, sus asociaciones $\mathrm{y}$ a diversos actores sociales que hoy se relacionan directa e indirectamente con la agricultura familiar santacruceña.

\section{LITERATURA CITADA}

ARIZPE, L. (1986). Las mujeres campesinas y la crisis agraria en América Latina, Mexico, Nueva Antropología, pp 1.

BIAGGI C., CANEVARI C., y TASSO A. (2007). Mujeres que trabajan la tierra. Un estudio sobre las mujeres rurales en la Argentina, Argentina, Serie estudio e investigaciones 11. Secretaría Agricultura, Ganadería, Pesca y Alimentos. Buenos Aires.

BIRGI, J., PERI, P. L., \& GARGAGLIONE, V. (2019). Raspberries and gooseberries in south Patagonia: Production, fruit quality, morphology and phenology in two environmental conditions. Scientia Horticulturae, 258, 108574.

BRUNDTLAND, GRO HARLEM, SUSANNA AGNELLI, SALEH ABDULRAHMAN ALATHEL, SALEH ABDULRAHMAN AL-ATHEL, PABLO GONZALEZ CASANOVA, BERNARD T. G. CHIDZERO, VOLKER HAUFF, ISTVAN LANG, MA SHIJUN, MARGARITA MARINO DO BOTERO, NAGENDRA SINGH, PAULO NOGUEIRA-NETO, SABURO OKITA, SHRIDATH S. RAMPHAL, WILLIAM DOYLE RUCKELSHAUS, MOHAMED SAHNOUN, EMIL SALIM, BUKAR SHAIB, VLADIMIR SOKOLOV, JANEZ STANOVNIK, MAURICE STRONG, JIM MACNEILL. (1987). Our common future ('brundtland reportl'), Noruega, General Assembly of the United Nations. nnn p.

CEPPARO, M. E. (2013). La Agricultura en la provincia de Santa Cruz.

FORO NACIONAL DE AGRICULTURA NACIONAL. Documento base del FONAF para implementar las políticas públicas del sector de la Agricultura Familiar (2006), Argentina, pp 9.

MALETTA H. (2011). Tendencias y perspectivas de la agricultura familiar en América Latina, Chile, Centro Latinoamericano para el Desarrollo Rural. pp 8. 
ORGANIZACIÓN DE LAS NACIONES UNIDAS PARA LA ALIMENTACIÓN Y LA AGRICULTURA. (2011). El estado mundial de la agricultura y la alimentación, las mujeres en la agricultura familiar, Italia, Disponible en: http://www.fao.org/docrep/013/i2050s/i2050s.pdf [Consultado el 26 de enero de 2018].

ORGANIZACIÓN MUNDIAL DE LA SALUD. (2002). Envejecimiento activo: un marco político, España. Traductor: Dr. Pedro J. Regalado Doña, pp 74.

LEONARDO, A. (2015). Manual del cálculo de la eficiencia de riego, Perú, Dirección General de Infraestructura y Riego. Ministerio de Agricultura y Riego. nnn p.

RENAF (Registro Nacional de la Agricultura Nacional) (2014). Caracterización estadística por región. Ministerio de Agricultura, Ganadería y Pesca, Argentina, Disponible en: https://www.renaf.magyp.gob.ar/documentos/InformeNacional2014.pdf [Consultado el 26 de enero de 2018].

SALCEDO, S., DE LA O A. P., GUZMÁN L. (2014). El concepto de agricultura familiar en América Latina y el Caribe. En: Salomón Salcedo y Lya Guzmán (Coord.). Agricultura Familiar en América Latina y el Caribe: Recomendaciones de Política. pp 26. Chile. Organización de las Naciones Unidaspara la Alimentación y la Agricultura. 